\title{
Evaluation of Mental Foramen Position from Panoramic Dental Radiographs
}

\author{
Muhammed Ajmal
}

\begin{abstract}
Objective: Evaluate the position of mental foramen by measuring the vertical bone height from digital panoramic radiograph and also the variation of resorption pattern by gender and extraction time frame.
\end{abstract}

Materials and methods/study design: Digital panoramic radiographs of 500 patients were reviewed. The study population was divided into four age groups aged 30 to 70 years as (30-39, 40-49, 50-59 and 60-70 years of age).

Radiographic Position of mental foramen was evaluated in each panoramic radiograph by measuring the distance from the marginal bone to the mental foramen was being measured.

Measurements were taken in each radiograph using Cliniview software. The data collected was subjected to statistical analysis using paired students t-test and chi-square tests.

Results and conclusion: In our study it was noted that the vertical measurements calculated from the superior margin of mental foramen to the crest of alveolar ridge were greater in edentulous men than in women. This measurement decreased significantly with age. We have noted here that resorption pattern between males and females can be used as an early diagnostic tool and for implant analysis. Studies comparing right and left side edentulous mandibular region are needed for further confirmation.

Keywords: Alveolar bone resorption, Mandibular Edentulous Posterior region, Mental Foramen, Panoramic radiography, Resorption pattern, Vertical bone height.

How to cite this article: Ajmal M. Evaluation of Mental Foramen Position from Panoramic Dental Radiographs. J Contemp Dent Pract 2014;15(4):399-402.

Source of support: Nil

Conflict of interest: None declared

\section{INTRODUCTION}

The loss of teeth causes remodeling and resorption of the surrounding alveolar bone and eventually leads to atrophic edentulous ridges. In edentulous patients, reduction of the

\footnotetext{
Assistant Professor

Division of Oral Diagnostic Sciences, College of Dentistry King Khalid University, Abha, Saudi Arabia

Corresponding Author: Muhammed Ajmal, Assistant Professor, Division of Oral Diagnostic Sciences, College of Dentistry, King Khalid University, Abha, Saudi Arabia, Phone: +966 567959512, e-mail: drmajmal@gmail.com
}

residual ridge is one of the most important factors affecting denture support, retention, stability and masticatory function. ${ }^{1}$

Mandibular edentulous arches provide one of the most needed radiological marker tools for diagnosis of various disorders as they have two most important anatomic landmarks mental foramen and mandibular canal which forms the basis in implant placement, forensic analysis and even studying the edentulous span after extraction due to the position of the mental foramen. Although distortion can be a major problem with panoramic radiographs, when performed properly they can provide valuable information, and are both readily accessible and cost efficient. ${ }^{2}$

The accurate identification of the mental foramen is important for both diagnostic and clinical procedures. In the past decades, X-rays have been widely used in dentistry. ${ }^{3}$ The ability to determine the quality and quantity of available bone in the potential implant site is paramount importance. Preimplant radiographs are important part of clinical evaluations before implant surgery. ${ }^{4-7}$ The purpose of this study was to evaluate the position of mental foramen by measuring the vertical bone height by using digital panoramic radiograph, also the variation of resorption pattern by gender and extraction time frame.

\section{MATERIALS AND METHODS}

On radiograph the mental foramen presents as a single circular or elliptical radiolucent area bilaterally in the premolar region. ${ }^{8}$ Several studies comparing different radiographic methods to assess the location of the mental foramen have suggested that digital panoramic radiographs show least error in the imaging of the mental foramen., ${ }^{9,10}$

Study design: In this descriptive study, a total of 500 panoramic radiographs of unilateral mandibular edentulous patient were examined. The age group selected was between 30 to 70 years. Patients were divided into four age groups as 30-39, 40-49, 50-59 and 60-70. Measurements were made from reference lines drawn from anatomic landmarks on standardized panoramic radiographs. All radiographs were made using a standardized manner by the same technician. The distance was measured from alveolar crestal bone to superior margin of mental foramen in edentulous sites by the aid of digital panoramic radiographs. The measurements 


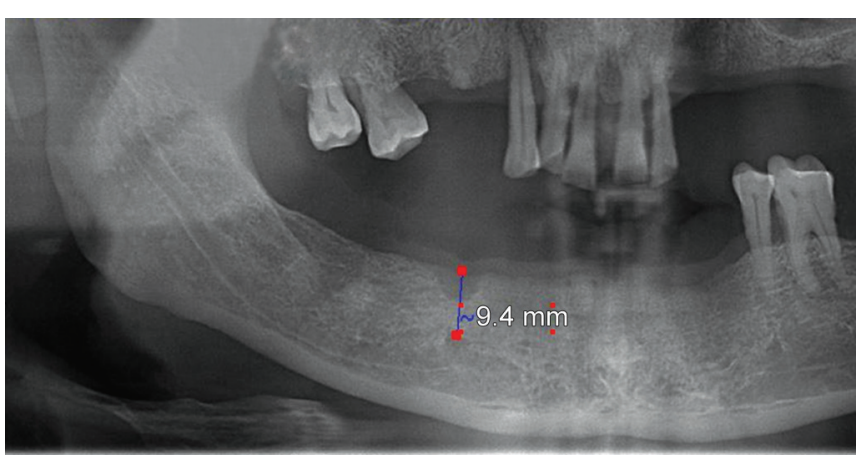

Fig. 1: Measurement from the marginal ridge to the mental foramen

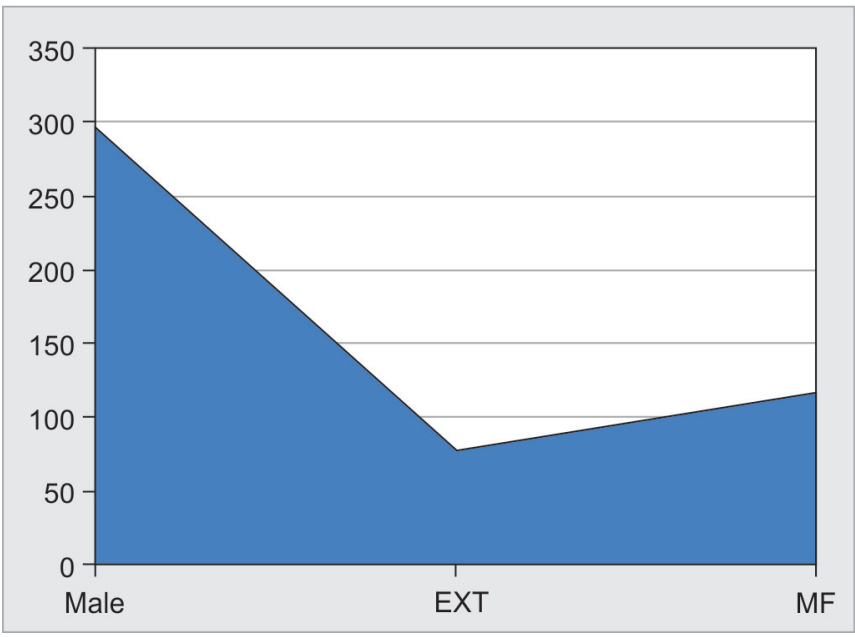

Graph 2: Resorption patterns in males and females

were done by using the ruler provided by the Clini-view software of Instrumentarium Company (Fig. 1). The magnification ratio of the panoramic Image was 1:1 as given by the manufacturer.

\section{RESULTS}

In Graph 1, a general distribution pattern was being analyzed with regard to males and females of the total radiographs evaluated for our study. The total numbers of males to females were almost the same. In Graphs 2 and 3, extractions were done differed from one another as the female subjects got their edentulous spans quite early compared to their male counterparts, most female extraction edentulous area correlations were between 50 and 59 years and males were between 60 and 70 . The varying reasons attributed are onset of osteoporosis, hormonal changes, loss of periodontal support, poor oral hygiene, etc.

Mental foramen in particular has been a useful marker of identification radiographically, as we know the resorption pattern varies significantly with age and edentulous time

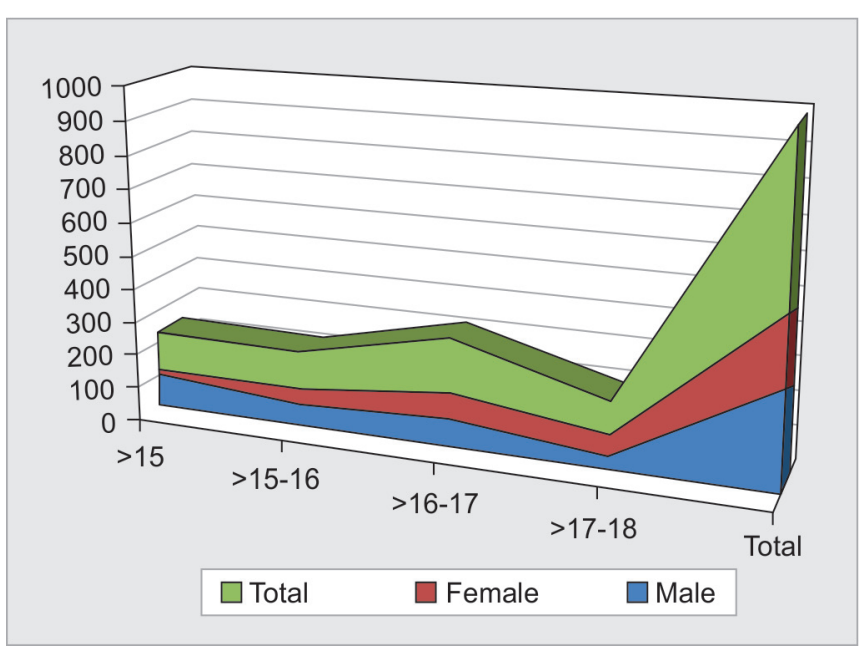

Graph 1: Distribution of males and females

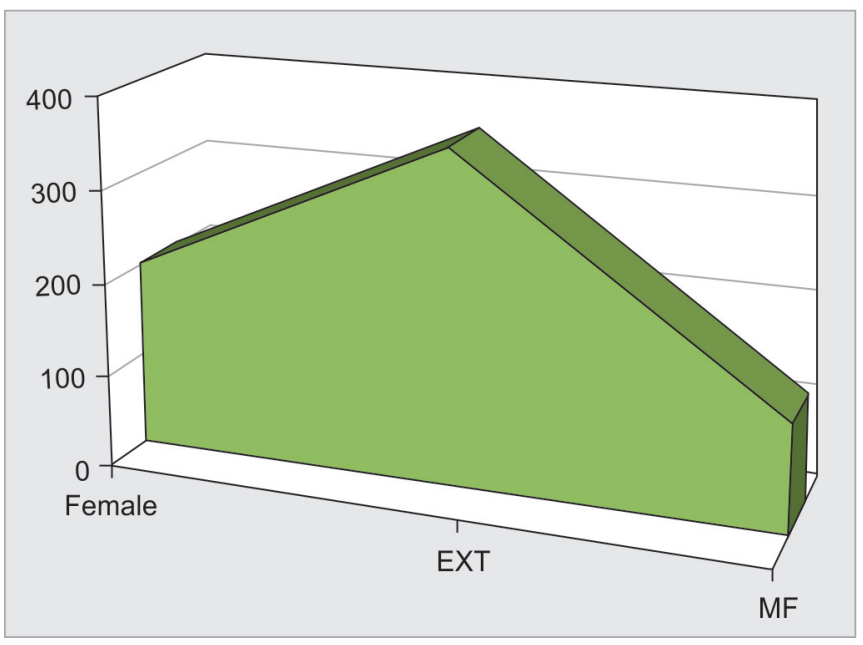

Graph 3: Comparison between extraction time frame and gender

span, a correlation was proposed between approximate time span between extraction and the existing edentulous state.

Anova analysis (Table 1) was done between the extraction time frame and the distance between marginal ridges to the mental foramen. The corelation between extraction time span and the distance between the crest of the alveolar ridge to the mental foramen decreases significantly in females. The resorption pattern in our study was rapid in females compared to males. This proves that distance between crest of alveolar ridge and superior margin of mental foramen may be used for gender and sex determination.

In Table 2 Pearson chi-square likelihood ratio and the tests like linear by linear association used for comparative analysis proves that with our given sample size the extraction time frame and gender resorption patterns was found to be more in females.

Table 3 (Mean $+\mathrm{SD}$ in $\mathrm{mm}$ ) shows distance between the superior foramina to crest of alveolar ridge $v s$ age with every age group there is a marginal difference between males and females, mean distance of the crestal margin to the mental 
Table 1: Extraction time frame and mental foramen

\begin{tabular}{|c|c|c|c|c|c|c|}
\hline \multicolumn{7}{|c|}{ ANOVA } \\
\hline & & Sum of Squares & $d f$ & Mean Square & $F$ & Sig. \\
\hline \multirow[t]{3}{*}{ EXT } & Between groups & 52.761 & 4 & 13.190 & 73.701 & 0.000 \\
\hline & Within groups & 88.591 & 495 & 0.179 & - & - \\
\hline & Total & 141.352 & 499 & - & - & - \\
\hline \multirow[t]{3}{*}{ MF } & Between groups & 361.996 & 4 & 90.499 & 216.409 & 0.000 \\
\hline & Within groups & 207.002 & 495 & 0.418 & - & - \\
\hline & Total & 568.998 & 499 & - & - & - \\
\hline
\end{tabular}

Table 2: Extraction time frame and gender (chi-square tests)

\begin{tabular}{llll}
\hline & Value & $d f$ & Asymp. Sig. (2-sided) \\
\hline Pearson chi-square & $241.597^{\mathrm{a}}$ & 9 & 0.000 \\
Likelihood ratio & 216.384 & 9 & 0.000 \\
Linear-by-Linear & 122.463 & 1 & 0.000 \\
Association & & & - \\
N of valid cases & 500 & - & - \\
\hline
\end{tabular}

Table 3: Distance between the superior foramina to crest of alveolar ridge vs age (Mean + SD in mm)

\begin{tabular}{lll}
\hline Age group (in years) & Difference in HT males $(\mathrm{mm})$ & Difference in HT females $(\mathrm{mm})$ \\
\hline $30-39$ & $20.4 \pm 3.3$ & $19.5 \pm 3.2$ \\
$40-49$ & $19.2 \pm 3.3$ & $18.7 \pm 3.3$ \\
$50-59$ & $18.6 \pm 1.3$ & $16.8 \pm 1.2$ \\
$60-70$ & $15.1 \pm 1.2$ & $13.2 \pm 1.2$ \\
\hline
\end{tabular}

foramen is increased in males compared to females and it is less pronounce between 30 and 50 years compared to patients who are more than 50 years of age.

Between the groups of in and above 50 years group: Statistical correlation showed a significant increase in the distance between superior margin of mental foramen from the alveolar crest in the male patients $(p<0.01)$ when compared with the female patients.

The period of extraction and the distance between superior margin of mental foramen to crest of alveolar ridge (SM to AR) decreases in females, and resorption were rapid in females compared to males.

All vertical measurements distance were significantly greater in the edentulous men than in the edentulous women $(p<005)$. In our study the decrease in the height of the edentulous mandible was more pronounced in women than in men.

Also, the distance between crest of alveolar ridge to superior margin of mental foramen (SM to SR) decreases significantly with age.

\section{DISCUSSION}

This study investigated whether or not a relationship exists between mandibular resorption and the length of edentulism period and/or age. Panoramic radiography is widely used and often used tool in routine dental diagnosis and prognosis. Especially implant treatment planning the panoramic radiographs are the most important criteria for implant selection.

Assessing residual ridge resorption, position of maxillary sinus, mandibular canal and mental foramen using panoramic radiographs are possible for examinations of large samples of patients. ${ }^{6}$ In all growing individuals the bone formation and bone resorption are simultaneously present; rather bone formation is slightly more than bone resorption. In adult the equilibrium of two processes can be noticed. But however, in the aged the resorption may not be compensated by production of bone resulting in senile osteoporosis. Thus, the bone resorption is a part of normal aging and is balanced between the anabolic and catabolic metabolism of the body. In postmenopausal women; deficiency of estrogen hormone accelerates skeletal bone loss and may result in rapid alveolar bone resorption. ${ }^{7}$

Gross resorption of the edentulous mandibular alveolar process resulting in excessive loss of denture bearing ridge is one of the most difficult restorative problems experienced by the prosthodontists. Several studies have evaluated age-related changes in mandibular bone. ${ }^{11}$ In this study the bone height measurement affected from gender of the population. Alveolar atrophy after the removal of teeth and the presence of tooth components in both the upper and lower jaws of edentulous patients has always been topic of discussion for dentists. ${ }^{12}$

This study attempted to test the usefulness of panoramic radiography in assessing bone height for implant recipient 
sites, to be used as a diagnostic tool for forensics and gender identification with resorption patterns. In our study the decrease in the height of the edentulous mandible was more pronounced in women than in men as observed by Ortman et $\mathrm{al}^{13}$ and Baat et al. ${ }^{14}$

Panoramic radiograph will have some amount of distortion, which will add to measurement. The amount of resorption can be well judged on panoramic images, when lower border of mental foramen and lower border of mandible are taken into consideration which is considered as lesser distortion areas. ${ }^{15}$

In postmenopausal women, deficiency of estrogen hormone accelerates skeletal bone loss and may result in rapid alveolar bone resorption. ${ }^{16}$ The findings obtained by orthopantomography (OPG) presented as mandibular indices can be used as early evidence of any changes in the total bone mineral density. These indices can be determined by the number of lost teeth and the alveolar bone resorption. ${ }^{17-19}$

Vertical heights of alveolar bone, localization of anatomic landmarks are important factors for planning of the implant supported prosthesis, forensic analysis and sex determination. The results of this study may guide clinicians to do so.

\section{CONCLUSION}

The mental foramen position is not constant and changes as age advances, there is also a gender related variation in the position of mental foramen in each age group we have studied. These variations in the position of the mental foramen have been reported to exhibit a clear trend in the pattern of their occurrence in relation to both age and gender wise. They form an important landmark in forensic analysis as well as in implant placement.

In microscopic pathology of edentulous ridges, there is an osteoclastic activity, especially on the external surface of the crest of residual ridges. Mandible having severe resorption may display gross porosity of the medullary bone on the crest of the ridge and may eventually uncover the mental foramen and the inferior alveolar canal. ${ }^{18}$

An accurate assessment of position of mental foramen and crest of alveolar ridge is very useful for prevention of postsurgical neurovascular complications and also holds the potential of contributing as an aid for forensic identification. Multicenteric studies with in different populations would help in establishing its role in forensic odontology.

\section{REFERENCES}

1. Soikkonen K, Ainamo A, Xie Q. Height of the residual ridge and radiographic appearance of bony structure in the jaws of clinically edentulous elderly people. J Oral Rehabil 1996;23(7): 470-475.
2. Garg AK, Vicari A. Radiographic modalities for diagnosis and treatment planning in implant dentistry. Implant Soc 1995;5(5): 7-11.

3. Ribeiro DA. Cytogenetic biomonitoring in oral mucosa cells following dental X-ray. Dentomaxillofac Radiol 2012;41(3): 181-184.

4. Kim YK, Park JY, Kim SG, Kim JS, Kim JD. Magnification rate of digital panoramic radiographs and its effectiveness for preoperative assessment of dental implants. Dentomaxillofac Radiol 2011;40(2):76-83.

5. Ozan O, Turkyilmaz I, Ersoy AE, McGlumphy EA, Rosenstiel SF. Clinical accuracy of 3 different types of computed tomography-derived stereolithographic surgical guides in implant placement. J Oral Maxillofac Surg 2009;67(2): 394-401.

6. Pompa V, Galasso S, Cassetta M, Pompa G, De Angelis F, Di Carlo S. A comparative study of Magnetic Resonance (MR) and Computed Tomography (CT) in the pre-implant evaluation. Ann Stomatol (Roma) 2010 Jul-Dec;1(3-4):33-38.

7. Saglam AA. The vertical heights of maxillary and mandibular bones in panoramic radiographs of dentate and edentulous subjects. Quintessence Int 2002;33(6):433-438.

8. Yosue T, Brooks SL. The appearance of mental foramina on panoramic and periapical radiographs. II. Experimental evaluation. Oral Surg Oral Med Oral Pathol 1989;68(4): 488-492.

9. Peker I, Gungor K, Semiz M. Localization of Mental and mandibular foramens on the conventional and digital panoramic images, Coll Antropol 2009;33(3):857-862.

10. Phillips JL, Weller RN, Kulild JC. The mental foramen: Part I. size, orientation, and positional relationship to the mandibular second premolar. J Endodont 1990 May;16(5):221-223.

11. Campbell RL. A comparative study of the resorption of alveolar ridges. JADA 1960;60:143-153.

12. Yamaoka M, Furusawa K, Fujimoto K, Uematsu T. Completely impacted teeth in dentate and edentulous jaws. Aust Dent J 1996; 41(3):169-172.

13. Ortman LF, Hausmann E, Dunford RG. Skeletal osteopenia and residual ridge resorption. J Prosthet Dent 1989;61(3): 321-325.

14. de Baat C, Kalk W, van 't Hof M. Factors connected with alveolar bone resorption among institutionalized elderly people. Community Dent Oral Epidemiol 1993;21(5):317-320.

15. Karaagaclioglu L, Ozkan P. Changes in mandibular ridge height in relation to aging and length of edentulism period. Int J Prosthodont 1994;7(4):368-371.

16. Devlin H, Ferguson MW. Alveolar ridge resorption and mandibular atrophy: a review of the role of local and systemic factors. Br Dent J 1991;170(3):101-104.

17. Klemetti E, Kolmakow S. Morphology of the mandibular cortex on panoramic radiographs as an indicator of bone quality. Dento-maxillofac Radiol 1997 Jan;26(1):22-25.

18. Klemetti E, Kolmakow S. Kroger H. Pantomography in assessment of the osteoporosis risk group. Scand J Dent Res 1994 Feb; 102(1):68-72.

19. Nakamoto T, Taguchi A, Ohtsuka M, Suei Y, Fujita M, Tanimoto $\mathrm{K}$, et al. Dental panoramic radiograph as a tool to detect postmenopausal women with low bone mineral density: untrained general dental practitioners' diagnostic performance. Osteoporos Int 2003 Aug;14(8):659-664. 